\title{
Approaches to Sensitivity Analysis in MOLP
}

\author{
Sebastian Sitarz \\ Institute of mathematics, University of Silesia, Poland \\ E-mail: ssitarz@math.us.edu.pl
}

\begin{abstract}
The paper presents two approaches to the sensitivity analysis in multi-objective linear programming (MOLP). The first one is the tolerance approach and the other one is the standard sensitivity analysis. We consider the perturbation of the objective function coefficients. In the tolerance method we simultaneously change all of the objective function coefficients. In the standard sensitivity analysis we change one objective function coefficient without changing the others. In the numerical example we compare the results obtained by using these two different approaches.
\end{abstract}

Index Terms - Multi-Criteria Decision Making, MultiObjective Linear Programming, Sensitivity Analysis

\section{Introduction}

\subsection{The aim of the Paper}

The paper analyses the sensitivity of the efficient solution in MOLP (and the weak efficient solutions). This problem was considered by many authors in different ways. The paper of Hansen et al. [1] was one of the first attempts to use the tolerance approach in the MOLP. Hladik [2] gave some approximation methods for the maximal tolerance. The approach based on the volume of the polyhedron was described by Vetchera [3]. Sitarz [4] gave a method of analysing the sensitivity approach in MOLP based on the standard sensitivity approach in linear programming.

In this work we present two approaches. The first of them serves finding a tolerance interval. This approach is used in the case of changing all the objective function coefficients. We present the approximation method for this approach, which is based on solving some additional linear programming problems. The other approach is used in the case of changing only one objective function coefficient. This approach is based on the parameterization of the test problem for efficiency given by Steuer [5]. In this case we also present the sensitivity of weak efficiency. The following papers on the sensitivity analysis in MOLP are worth mentioning: the range set approach by Benson [6], the interval coefficients by Chanas and Kuchta [7], the tolerance approach by Hladik [8], standard sensitivity approach in MOLP by Sitarz $[9,10$, 11]

\subsection{Outline of the paper}

This paper consists of the following sections: section 2 presents the introduction to MOLP, section 3 considers the additive tolerance approach to MOLP, section 4 analyses the standard sensitivity analysis to MOLP (this section is a continuation of previous works by Sitarz [4, 12]). Next sections give the numerical example and application which illustrate the presented theory. The last section summarizes the paper

\section{Notation}

\subsection{Main Notation}

The notation used in the paper is as follows. We consider multi-objective linear programming problem in the following form:

$$
\operatorname{VMax}\{\boldsymbol{C x}: \boldsymbol{x} \in X\}
$$

where

$X=\left\{\mathbf{x} \in R^{n}: \mathbf{A x} \leq \mathbf{b}, \mathbf{x} \geq 0\right\}$ or $X=\left\{\mathbf{x} \in R^{n}: \mathbf{A x}=\mathbf{b}, \mathbf{x} \geq 0\right\}$ feasible region in decision space.

$\mathbf{x} \quad \mathrm{R}^{n}$ - vector of decision variables

C $\mathrm{R}^{n, k}$ - matrix of objective function coefficients

A $\mathrm{R}^{n, m}$-full row rank matrix of constraint coefficients

b $\quad \mathrm{R}^{m}$ - right hand side vector

\subsection{Degenerated Solutions}

For the basic solution of problem (1) connected with the base $\left\{\mathbf{a}^{j_{1}}, \mathbf{a}^{j_{2}}, \ldots, \mathbf{a}^{j_{m}}\right\}$ we will use the following notation:

$\mathrm{B}=\left\{j_{1}, \ldots, j_{m}\right\}-$ index set of the base

$\mathbf{A}_{\mathrm{B}}=\left[\mathbf{a}^{j_{1}}, \mathbf{a}^{j_{2}}, \ldots, \mathbf{a}^{j_{m}}\right]$ - basic columns of $\mathbf{A}$,

$\mathbf{A}_{\mathrm{N}}$ - nonbasic columns of $\mathbf{A}$

$\mathbf{x}=\left[\mathbf{x}_{\mathrm{B}}, \mathbf{x}_{\mathrm{N}}\right]-$ basic solution associated with $\mathrm{B}$, $\left(\mathbf{x}_{\mathrm{B}}=\mathbf{A}_{\mathrm{B}}{ }^{-1} \mathbf{b} \geq \mathbf{0}, \mathbf{x}_{\mathrm{N}}=\mathbf{0}\right)$

$\mathbf{C}_{\mathrm{B}}\left(\mathbf{C}_{\mathrm{N}}\right)$ - basic (nonbasic)columns of $\mathbf{C}$

$\overline{C_{N}}$ - matrix of the reduced costs 
For the degenerated basic solution of (1) we will use the following notation:

$\left(x_{B}\right)_{i}=0$ - degenerated basic variable

$d$ - number of degenerated basic variables

$\left[\mathbf{A}_{B}^{-1} \mathbf{A}_{N}\right]_{D}$ - matrix associated with degenerated basic variables and nonbasic column.

For the extreme point of $\mathrm{X}$ we define the polar cone of $\mathrm{X}$ at the point $\mathrm{X}^{*}$ in the following way:

$$
N\left(x^{*}\right)=\left\{x^{*} \in \boldsymbol{R}: H x \geq 0\right\}
$$

where $H=\left[-h_{i}\right]_{i \in I}$ and $h_{i} \in \boldsymbol{R}$ are all vectors of the directions for the edges $(i \in I)$ of $X$ emerging from $\mathrm{x}^{*}$. In the case of the nondegenerated basic solution $x=\left[x_{B}, x_{N}\right]$, we have

$$
H=\left\lfloor\left(A_{B}^{-1}\right)^{T}, 0\right\rfloor
$$

We call $\mathbf{x} \in \mathrm{X}$ the efficient solution of (1) if

$$
\sim \exists_{\mathrm{x}^{\prime} \in \mathrm{X}}: \quad \mathbf{C} \mathbf{x}^{*} \leq \mathbf{C} \mathbf{x}^{\prime} \wedge \mathbf{C x}^{*} \neq \mathbf{C} \mathbf{x}^{\prime}
$$

We call $\mathbf{x} \in X$ the weak efficient solution of (1) if

$$
\sim \exists_{\mathrm{x}^{\prime} \in \mathrm{X}}: \quad \mathbf{C x}^{*}<\mathbf{C} \mathbf{x}^{\prime}
$$

It is obvious that every efficient solution is the weak efficient solution.

\section{Additive Tolerance}

The additive tolerance approach aims at finding a value (tolerance) representing the maximum absolute additive perturbation which can be applied simultaneously without effecting the efficiency. The presented results are based on works [1, 2].

Let us introduce an additive $\delta$-neighbourhood of a matrix $\mathbf{C}=\left[c_{i j}\right]$ :

$$
O_{\delta}(C)=\left\{D=\left[d_{i j}\right]: \max _{i, j}\left|d_{i j}-c_{i j}\right| \leq \delta\right\}
$$

We consider a new problem:

$$
\operatorname{VMax}\{\boldsymbol{D} \boldsymbol{x}: \boldsymbol{x} \in X\}
$$

which is built from problem (1) by changing matrix C by matrix $\mathbf{D}$.

\section{Definition 1.}

An additive tolerance for an efficient solution $x^{*}$ is any real $\delta>0$ such that $x^{*}$ remains efficient to (2) for all
$D \in O_{\delta}^{+}(C)$. A maximal additive tolerance we denote as $\delta_{\max }$.

Remark 1 [methods for computing the maximal tolerance]

Algorithms for computing the maximal tolerance can be found in the work by Hansen et al. [1].

The work by Hladik [3] gives the approximation method for finding $\delta_{\max }$. In the following theorem, we use the following notation:

$$
\begin{aligned}
& |H|=\left\lfloor h_{i j} \mid\right\rfloor_{i j}-\text { matrix of the absolute values of } h_{i j} . \\
& \mathbf{1} \in \boldsymbol{R}^{m}-\text { vector of ones. }
\end{aligned}
$$

\section{Theorem 1. [2]}

Let $\left(\lambda_{\mathrm{opt}}, \delta_{\mathrm{opt}}\right)$ be the optimal solution to the following program:

$\operatorname{Max} \delta$

$$
\begin{gathered}
H C^{\mathrm{T}} \lambda-|H| \mathbf{1} \delta \leq 0 \\
\mathbf{1}^{\mathrm{T}} \lambda=1 \\
0 \leq \lambda \in \boldsymbol{R}^{m}, 0 \leq \delta \in \boldsymbol{R}
\end{gathered}
$$

Then $\delta_{\text {opt }}$ is an additive tolerance for problem (2).

\section{Remark 2. [to theorem 1]}

Problem (3) is a linear programming problem, which makes it easy to solve. Moreover, it is obvious that $\delta_{\text {opt }}<\delta_{\max }^{+}$, but we do not know how much $\delta_{\text {opt+ }}$ may differ from $\delta_{\max }^{+}$, (in [2] we cannot find the upper limit of this approximation).

\section{Standard Sensitivity Analysis}

\subsection{Main results}

The standard sensitivity analysis aims at finding the values (a parameter set) of one objective function coefficient which can be applied without effecting the efficiency. The presented results are based on Sitarz [4, $12]$.

Let us introduce a new matrix $\mathbf{D}_{t}=\left[d_{k l}\right]$ which is obtained from matrix $\mathbf{C}$ by changing only one element $c_{i j}$ into parameter $t$ :

$$
d_{k l}= \begin{cases}c_{k l}, & \text { if }(k, l) \neq(i, j) \\ t, & \text { if }(k, l)=(i, j)\end{cases}
$$


We consider a new problem:

$\operatorname{VMax}\left\{\boldsymbol{D}_{t} \boldsymbol{x}: \boldsymbol{x} \in X\right\}$

\section{Definition 2.}

a. The set of all parameters $t$, for which $x^{*}$ is the weak efficient solution to (4) will be denoted by $T_{x}^{W}$.

b. The set of all parameters $t$, for which $x^{*}$ is the efficient solution to (4) will be denoted by $T_{x}$.

\subsection{Topological Properties}

The topological properties of sets $T_{x^{*}}$ and $T_{x^{*}}^{W}$ are given in the following theorems.

Theorem 2. [4, 12]

a. The set $T_{x}$, is an interval.

b. The set $T_{x}^{W}$ is a closed interval.

\subsection{Methods of computation}

We present the methods for describing sets $T_{r}$, and $T_{x}^{W}$. In the following theorem, we use the following notation:

$I \in \boldsymbol{R}^{d x d}$ - vector of ones,
$\mathbf{1} \in \boldsymbol{R}^{n-m}$ - identity matrix.
$\overline{D_{N}}$ - reduced cost matrix to problem (4) associated with nonbasic columns

\section{Theorem 3. [5]}

a. The solution $\mathbf{x}^{*}$ is efficient to problem (4) if and only if the problem:

$$
\begin{aligned}
& \operatorname{Max} r \\
& -\overline{D_{N}} y+\mathbf{1} r \leq 0 \\
& {\left[A^{-1}{ }_{B} A_{N}\right]_{d} y+I s=0} \\
& 0 \leq y \in \boldsymbol{R}^{n-m}, 0 \leq s \in \boldsymbol{R}
\end{aligned}
$$

has a bounded objective function value of zero.

b. The solution $\mathbf{x}^{*}$ is weak efficient to problem (4) if and only if the problem:
Max $r$

$$
\begin{aligned}
& -\overline{D_{N}} y+\mathbf{I} v=0 \\
& {\left[A^{-1}{ }_{B} A_{N}\right]_{d} y+\mathbf{I} s=0} \\
& 0 \leq y \in \boldsymbol{R}^{n-m}, 0 \leq v \in \boldsymbol{R}^{k}, 0 \leq s \in \boldsymbol{R}
\end{aligned}
$$

has a bounded objective function value of zero.

Remark 3. [methods for finding the sets $T_{x}$, and $T_{x^{*}}^{W}$ ]

The detailed description of the algorithm for finding sets $T_{x}$, and $T_{x}^{W}$ are given in works $[4,12]$. Here, these methods are described in general way. As you may notice parameterizing the coefficient $c_{i j}$ in the problem (4) causes parameterizing constraint matrix in problems (5) and (6). Thus, initial multi-criteria problem (4) is transformed into one criterion problems (5) and (6) with the parameterized coefficient matrix. The sensitivity analysis of such one-criterion problems was presented in a detailed way in Gal [13]. The results of this analysis were used in Sitarz [6]. In this paper you can find the detailed computation. It is worth to notice the paper by Hladik and Sitarz [14], where some methods for computing are presented.

\section{Numerical Example}

Consider the following problem $[15,2]$ :

$$
\begin{aligned}
& \operatorname{VMax}\left[\begin{array}{l}
2.5 x_{1}+2 x_{2} \\
3.5 x_{1}+0.65 x_{2}
\end{array}\right] \\
& 3 x_{1}+4 x_{2} \leq 42 \\
& 3 x_{1}+x_{2} \leq 24 \\
& x_{2} \leq 9
\end{aligned}
$$

The set of feasible solutions $\mathrm{X}$ is a polyhedron with the following extreme points:

$$
x^{1}=[0,9], x^{2}=[2,9], x^{3}=[6,6], x^{4}=[8,0], x^{5}=[0,0]
$$

The set of all efficient solutions contains one edge: $\overline{x_{3} x_{4}}$, this edge presents the set of all weak efficient solutions, too. Fig. 1 presents the graphical illustration of this problem.

Let us analyse the sensitivity for extreme points $x^{3}=[6,6]$ and $x^{4}=[8,0]$ by using the approaches presented earlier. The results are presented in Table 1. 
Table 1: The additive tolerance (approximated and maximal) and the intervals obtained by using the standard sensitivity analysis

\begin{tabular}{|c|c|c|}
\hline$\delta_{\mathrm{opt}} ; \delta_{\max }$ & $\begin{array}{c}x^{3} \\
0.716 ; \quad 0.875\end{array}$ & $\begin{array}{c}x^{4} \\
0.3875 ; \quad 0.3875\end{array}$ \\
\hline \multicolumn{3}{|c|}{$c_{11}=2.5$} \\
\hline$T_{x^{1}}$ & $(-\infty, 6)$ & $(-\infty,+\infty)$ \\
\hline$T_{x^{1}}^{W}$ & $(-\infty, 6]$ & $(-\infty,+\infty)$ \\
\hline$\left[c_{11}-\delta_{\max }, c_{11}+\delta_{\max }\right]$ & {$[1.6,3.3]$} & {$[2.1,2.8]$} \\
\hline \multicolumn{3}{|c|}{$c_{12}=2$} \\
\hline$T_{x^{1}}$ & $(0.833,+\infty)$ & $(-\infty,+\infty)$ \\
\hline$T_{x^{1}}^{W}$ & {$[0.833,+\infty)$} & $(-\infty,+\infty)$ \\
\hline$\left[c_{12}-\delta_{\max }, c_{12}+\delta_{\max }\right]$ & {$[1.1,2.8]$} & {$[1.6,2.3]$} \\
\hline \multicolumn{3}{|c|}{$c_{21}=3,5$} \\
\hline$T_{x^{1}}$ & $(-\infty,+\infty)$ & $(1.950,+\infty)$ \\
\hline$T_{x^{1}}^{W}$ & $(-\infty,+\infty)$ & {$[1.950,+\infty)$} \\
\hline$\left[c_{21}-\delta_{\max }, c_{21}+\delta_{\max }\right]$ & {$[2.625,4.375]$} & {$[3.1125,3.8875]$} \\
\hline \multicolumn{3}{|c|}{$c_{22}=0,65$} \\
\hline$T_{x^{\prime}}$ & $(-\infty,+\infty)$ & $(-\infty, 1.167)$ \\
\hline$T_{x^{1}}^{W}$ & $(-\infty,+\infty)$ & $(-\infty, 1.167]$ \\
\hline$\left[c_{22}-\delta_{\max }, c_{22}+\delta_{\max }\right]$ & {$[-0.2,1.5]$} & {$[0.2,1.0]$} \\
\hline
\end{tabular}

Remark 4 [to the results obtained in Table 1]

a) Comparing the intervals $T_{x^{\prime}} T_{x^{1}}^{W}$ with the intervals $\left[c_{\mathrm{ij}}-\delta_{\max }, c_{\mathrm{ij}}+\delta_{\max }\right]$ we can observe that the intervals $T_{x^{\prime}} T_{x^{1}}^{W}$ are bigger then $\left[c_{\mathrm{ij}}-\delta_{\max }, c_{\mathrm{ij}}+\delta_{\max }\right]$. This results from the way of the perturbation. In the first method, connected with $T_{x^{1}} T_{x^{\prime}}^{W}$, we change only one coefficient, although in the second method we change all the coefficients simultaneously and independently.

b) Let us notice that in all of the cases the closure of $T_{x^{\prime}}$ equals to $T_{x^{1}}^{W}$, this is not a rule, the sets $\mathrm{cl} T_{x^{\prime}}$ and $T_{x^{1}}^{W}$ can differ much more, Sitarz [12].

c) The values of $\delta_{\max }$ and its approximation $\delta_{\text {opt }}$ do not differ much. However, we do not know how much different they can be, Hladik [2].

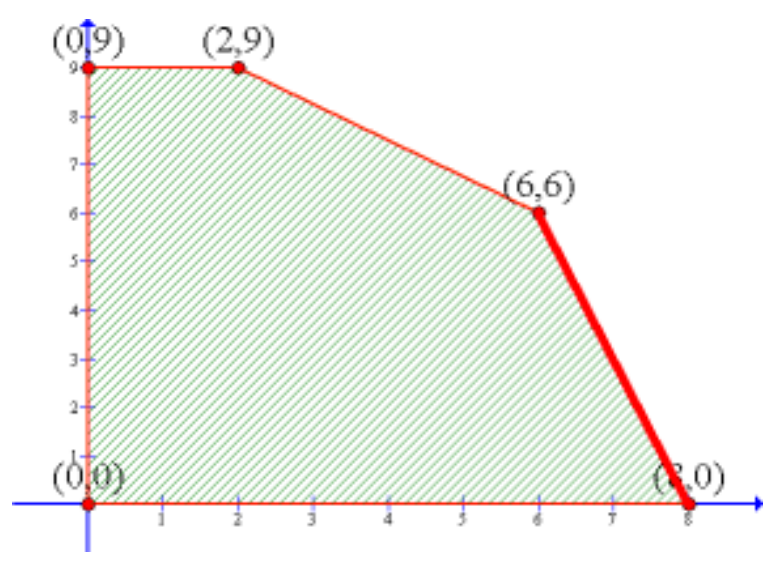

Fig. 1: Graphical illustration of the example in the decision space

\section{Application in diet problem}

We consider a famous problem of linear programming - the diet problem. This problem is analysed in the case of one-criterion in many works, for example by Gass [16, 17]. In the case of bi-criteria linear programming problem, the diet problem is considered by Benson and Morin [18] for the nutrition planning in developing nations. 
Here, we focus on the "Optimal Nutrition" proposed by Kwasniewski [19]. There are strict rules on the proportion between the three main food components: protein $(P)$, fat $(F)$ and carbohydrates $(C)$. The ideal proportion between the main food components should be in the range of :

$$
1(P) \quad: \quad 2.5-3,5(F): 0.5-0.8(C)
$$

Moreover, the correct amount of protein to be consumed in a day is $1 \mathrm{~g}$ per $1 \mathrm{~kg}$ of a bodyweight $(B W)$. In our model $(B W)$ is a constant parameter, thus we denote it by const ${ }_{B W}$. By using the above rules we take into account two criteria:

- minimizing the cost of the diet

- maximizing the food energy

To formulate MOLP problem we need to use some food products with known contents of protein, fat, carbohydrates and food energy. To illustrate this numerically, let us consider ten basic food products:

- Butter $-\mathrm{x}_{1}$

- Bacon $-\mathrm{x}_{2}$

- Chocolate $-\mathrm{x}_{3}$

- Cheese $-\mathrm{x}_{4}$

- Sour cream - $\mathrm{x}_{5}$

- Eggs - $\mathrm{X}_{6}$

- Salmon - $\mathrm{x}_{7}$

- Pasta $-\mathrm{x}_{8}$

- Bread - $\mathrm{X}_{9}$

- Potatoes - $\mathrm{x}_{10}$

We use the table of mean contents of $P, F, C(\mathrm{~g})$ and food energy (Kcal) (in $100 \mathrm{~g}$ of a given product) which can be found in a cookery book. Moreover, we proceed calculation for the mean costs of products in Poland (in 2012 for $100 \mathrm{~g}$ in euro) and we take const ${ }_{B W}=70$..

Now, we are able to present MOLP problem describing the above problem.

$$
\begin{aligned}
& \operatorname{Min} .60 x_{1}+.67 x_{2}+.48 x_{a}+ \\
& .60 x_{4}+.17 x_{5}+.41 x_{6}+.84 x_{7}+.17 x_{9}+ \\
& .13 x_{9}+.09 x_{10}
\end{aligned}
$$

$$
\begin{aligned}
& \operatorname{Max} 748 x_{1}+405 x_{2}+560 x_{9}+282 x_{4}+ \\
& 186 x_{5}+140 x_{6}+129 x_{7}+ \\
& 368 x_{9}+250 x_{9}+66 x_{10}
\end{aligned}
$$$$
P=14 x_{2}+6 x_{a}+26 x_{4}+3 x_{5}+
$$

$$
\begin{aligned}
& 11 x_{6}+13 x_{7}+12 x_{9}+6 x_{9}+x_{10} \\
& F=82 x_{1}+38 x_{2}+35 x_{9}+ \\
& 18 x_{4}+18 x_{5}+10 x_{6}+9 x_{7} \\
& C=60 x_{a}+4 x_{5}+75 x_{9}+55 x_{9}+15 x_{10} \\
& 2.5 P \leq F \leq 3.5 P \\
& 0.5 P \leq C \leq 0.8 P \\
& P=\text { const } t_{\text {BW }}=70 \\
& x_{1, \ldots g} x_{10} \geq 0
\end{aligned}
$$

We analyse the sensitivity of the following efficient solution:

$$
\begin{aligned}
& x_{4}^{*}=161 g, x_{5}^{*}=811 g, x_{g}^{*}=31 g, \\
& x_{1}^{*}=x_{2}^{*}=x_{g}^{*}=x_{6}^{*}=x_{7}^{*}=x_{9}^{*}=x_{10}^{*}=0
\end{aligned}
$$

It is worth mentioning that the values of criteria functions for this solution are as follows: 2,37 (cost), 2079 (energy) .

We present the results of sensitivity analysis by means of the additive tolerance approach. By using the above data, we obtain that the given solution $x^{*}$ remains efficient with changing simultaneously the cost coefficients maximally by $\delta_{\max }=9,4 \%$. In other words, the maximal additive tolerance for this solution is equal to 0.094 . It means that the coefficients of the objective functions for cost can be maximally changed by $9,4 \%$ and our solution is still efficient.

\section{Summary}

We have dealt with the sensitivity analysis of efficiency in MOLP. We analysed the perturbation of the objective function coefficients. We have presented two approaches: the tolerance approach and standard sensitivity analysis. On the basis of these approaches the feasible intervals of the coefficient perturbations were constructed (see Table 1). By comparing these intervals, big differences between the considered methods were observed, which was the consequence of the objective function coefficients' perturbation.

The future research can be directed into the method of connecting these two approaches and finding the mathematical relationships. It might be possible to find a new approach which can be a mean of these two approaches (the intervals between the intervals obtained by using these two methods). Moreover, the further research and problems to solve, according to the author, are as follows:

- numerical analysis of algorithms by using various optimization methods: [20], [21]. 
- extension using the augmented Tchebycheff metric: [22].

- comparison with outranking methods based on compromise programming: [23], [24].

- including more general structure to describe the preferences of decision maker, for instance fuzzy numbers or stochastic dominance: [25], [26].

\section{References}

[1] Hansen P., Labbe M., Wendell R.E. (1989). Sensitivity Analysis in Multiple Objective Linear Programming: The Tolerance Approach. European Journal of Operational Research 38, 63-69.

[2] Hladik M. (2008). Additive and multiplicative tolerance in multiobjective linear programming, Operations Research Letters 36, 393-396.

[3] Vetschera R. (1997). Volume-Based Sensitivity Analysis for Multi-Criteria Decision Models. In: Göpfert A., Seeländer J., Tammer Chr. (Eds.). Methods of Multicriteria Decision Theory. HänselHohenhausen, Egelsbach.

[4] Sitarz S. (2008). Postoptimal analysis in multicriteria linear programming. European Journal of Operational Research, 191, 7-18.

[5] Steuer R. (1986). Multiple Criteria Optimization Theory: Computation and Application. John Willey, New York.

[6] Benson H. P. (1985). Multiple objective linear programming with parametric criteria coefficients. Management Science 31 (4), 461-474.

[7] Chanas, S., Kuchta, D. (1996), Multiobjective programming in optimization of interval objective functions a generalized approach, European Journal of Operational Research 94, 594-598.

[8] Hladik M. (2008). Computing the tolerances in multiobjective linear programming. Optimization. Methods \& Software, 23 (5), 731-739.

[9] Sitarz S. (2010). Standard sensitivity analysis and additive tolerance approach in MOLP. Annals of Operations Research, 181(1), 219-232.

[10] Sitarz S. (2012). Mean value and volume-based sensitivity analysis for Olympic rankings, European Journal of Operational Research, 216, 232-238.

[11] Sitarz S. (2013). Parametric LP for sensitivity analysis of efficiency in MOLP problems, Optimization Letters, [in press], doi: 10.1007/s11590-012-0541-1.

[12] Sitarz S. (2011). Sensitivity analysis of weak efficiency in multiple objective linear programming. Asia-Pacific Journal of Operational Research, 28/4, 445-455.
[13] Gal T. (1995). Postoptimal Analyses, Parametric Programming and Related Topics. Walter de Gruyter, Berlin.

[14] Hladik M., Sitarz S., Maximal and supremal tolerances in multiobjective linear programming, European Journal of Operational Research, 228 (1), 93-101, 2013

[15] Oliveira C., Antunes C. H. (2007). Multiple objective linear programming models with interval coefficients - an illustrated overview, European Journal of Operational Research 181/3, 1434-1463.

[16] Gass S. I. (1970). An illustrated guide to linear programming. Mc Graw-Hill Book Company, New York.

[17] Gass S. I. (1975). Linear Programming, Methods and Applications. Mc Graw-Hill Book Company, New York.

[18] Benson H., Morin T. L. (1987). A bicriteria mathematical programming model for nutrition planning in developing nations. Managemant Science 33 (12), 1593-1601.

[19] Kwasniewski J., (1999). Optimal Nutrition. WGP, Warsaw.

[20] Sitarz S. (2006), Hybrid methods in multi-criteria dynamic programming, Applied Mathematics and Computation, 180/1, 38-45.

[21] Sitarz S. (2009). Ant algorithms and simulated annealing for multicriteria dynamic programming, Computers \& Operations Research, 36 (2), 433441.

[22] Steuer R., Choo E (1981). An Interactive weighted Tchebycheff procedure for multiple objective programming, Mathematical Programming 26, 326-344.

[23] Opricovic S., Tzeng G.H. (2004). Compromise solution by MCDM methods: A comparative analysis of VIKOR and TOPSIS, European Journal of Operational Research, 156, 445-455.

[24] Opricovic S., Tzeng G.H. (2007). Extended VIKOR method in comparison with outranking methods, European Journal of Operational Research, 178 (2), 514-529.

[25] Sitarz S. (2010). Dynamic Programming with Ordered Structures: Theory, Examples and Applications, Fuzzy Sets and Systems, 161, 26232641.

[26] Trzaskalik T., Sitarz S. (2007). Discrete dynamic programming with outcomes in random variable structures, European Journal of Operational Research, 177 (3), 1535-1548. 


\section{Author's Profiles}

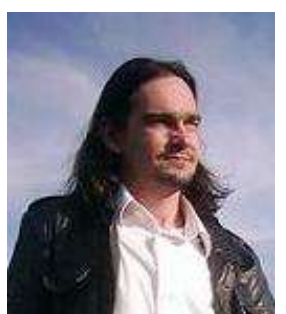

Sebastian Sitarz was born on March 19, 1975. He received his MS in Mathematics from University of Silesia (Poland) in 1999; PhD in Economics from University of Lodz in 2003 (Poland). Currently, he is working as a Lecturer in the Institute of Mathematics, the University of Silesia, Katowice (Poland), since 2003. His research areas include multiobjective linear programming, dynamic programming and their applications. His papers appeared in European Journal of Operational Research, Fuzzy Sets and Systems, Computers and Operations Research, Annals of Operations Research, Applied Mathematics and Computation, Asia Pacific Journal of Operational Research and others.

How to cite this paper: Sebastian Sitarz,"Approaches to Sensitivity Analysis in MOLP", International Journal of Information Technology and Computer Science(IJITCS), vol.6, no.3, pp.54-60, 2014. DOI: 10.5815/ijitcs.2014.03.07 